\title{
Evanescent modes in sonic crystals: Complex dispersion relation and supercell approximation
}

\author{
V. Romero-García, ${ }^{1,2, a)}$ J. V. Sánchez-Pérez, ${ }^{1}$ and L. M. Garcia-Raffi ${ }^{3}$ \\ ${ }^{1}$ Centro de Tecnologías Físicas: Acústica, Universidad Politécnica de Valencia, Camino de Vera s/n, 46022, \\ Valencia, Spain \\ ${ }^{2}$ Instituto de Ciencia de Materiales, Consejo Superior de Investigaciones Científicas. Sor Juana Inés de la \\ Cruz, 3, Cantoblanco, 28049, Madrid, Spain \\ ${ }^{3}$ Instituto Universitario de Matemática Pura y Aplicada, Universidad Politécnica de Valencia, Camino de \\ Vera $s / n$, 46022, Valencia, Spain
}

(Received 26 February 2010; accepted 26 June 2010; published online 20 August 2010)

\begin{abstract}
Evanescent modes in complete sonic crystals (SCs) and SC with point defects are reported both theoretically and experimentally in this paper. Plane wave expansion (PWE) and in general, $\omega(k)$ methods have been used to calculate band structures showing gaps that have been interpreted as ranges of frequencies where no real $k$ exists. In this work, we extend PWE to solve the complex $k(\omega)$ problem applied to SC, introducing the supercell approximation for studying one vacancy. Explicit matrix formulation of the equations is given. This $k(\omega)$ method enables the calculation of complex band structures, as well as enabling an analysis of the propagating modes related with real values of the function $k(\omega)$, and the evanescent modes related with imaginary values of $k(\omega)$. This paper shows theoretical results and experimental evidences of the evanescent behavior of modes inside the SC band gap. Experimental data and numerical results using the finite elements method are in very good agreement with the predictions obtained using the $k(\omega)$ method. (C) 2010 American Institute of Physics. [doi:10.1063/1.3466988]
\end{abstract}

\section{INTRODUCTION}

The propagation of scalar waves inside periodic structures has been receiving growing interest in recent years. A great effort has been made to understand the physics of these systems since the acoustical properties of a periodic sculpture by Eusebio Sempere were measured. ${ }^{1}$

Phononic crystals (PCs) consist of an inhomogeneous periodic distribution of elastic materials embedded in other elastic materials with different properties. ${ }^{2,3}$ These systems are extensions of the photonic crystals ${ }^{4,5}$ used for the propagation of elastic waves through periodic elastic structures. If one of the elastic materials is a fluid medium, then PCs are called sonic crystals (SCs). Several studies discuss the similarities and differences between these periodic systems. 6,7

The periodicity of these systems is introduced in the solution of the wave equation by means of Bloch's theorem. This solution leads to the phenomenon of band gaps (BGs): frequency regimes where waves do not propagate through the crystal. Traditionally, wave propagation inside such systems was analyzed by means of the band structures. Plane wave expansion (PWE) (Ref. 8) transforms the wave equation into an eigenvalue problem that can be solved for each Bloch vector, $k$, in the irreducible first Brillouin zone; and so obtaining the eigenfrequencies $\omega(\vec{k})$ that constitute the band structures. In the case of SCs, it has been proven that eigenfrequencies for an arbitrary crystal structure and an arbitrary filling fraction ${ }^{9}$ are real values. A great number of applications based on SCs are explained by the existence of BGs: acoustic filters; ${ }^{10}$ acoustic barriers; ${ }^{11}$ or wave guides. ${ }^{12,13}$

${ }^{a)}$ Electronic mail: virogar1@mat.upv.es.
Propagating waves inside a periodic media represent a set of solutions to the wave equation that satisfy the translational symmetry and these are characterized by the transmission bands in the PWE method. However, where the translational symmetry is broken, finite periodic media or periodic media with point defects, can support the well known evanescent modes characterized by a complex wave number, $k^{23}$ Recent experimental results ${ }^{14}$ show measurements of the sound levels recorded inside a point defect and behind an SC. These authors observed that this level is higher inside the cavity than behind the crystal. This fact clearly shows both the generation of a trapping mode (i.e., localized mode) inside the point defect and its evanescent behavior outside the vacancy. Some authors in the electromagnetic regime have measured the evanescent modes in photonic crystals and revealed multiexponential decay. ${ }^{15}$

Several extensions of the PWE method have been used to analyze the propagation of sound through periodic systems in different situations; for example, crystals with point defects have been analyzed with PWE using the supercell approximation. ${ }^{16,17}$ The same methodology has been used to analyze the influence of the following: constituent materials, plate thickness, and the geometry of the array on the band structure in two-dimensional (2D) PC plates. ${ }^{18}$ However, these $\omega(\vec{k})$ methods interpret the $\mathrm{BG}$ as frequency regimes where no real $k$ exists. Therefore, these methods can only be used to study and characterize propagating modes.

We have been motivated by the work of Hsue et al. ${ }^{19}$ in which the PWE was extended for the case of photonic crystals to calculate the complex $k$ in a $2 \mathrm{D}$ isotropic and in general three-dimensional (3D) anisotropic cases. In this paper we show the extended PWE (EPWE) for the case of 2D SCs. 
The aim is to obtain the band structures using the inverse expression $k(\omega)$, and with a possibly complex $k$. Recent works show the calculation of complex band structures for PCs. ${ }^{20,21}$ In the present work we show the explicit matrix formulation and the approximation of supercell for analyzing the complex relation dispersion of SCs. The extension of the methodology enables us to characterize the evanescent and propagating modes in complete SCs as well as in SCs with point defects.

In this paper we present novel measurements of the pressure in the space between rows inside an SC. We have developed a 3D computer-controlled automatic positioning system together with an automatized acquisition system, called 3DReAMS (3D Robotized e-Acoustic Measurement System). This system enables the pressure field in trajectories inside a crystal to be measured and we have consequently analyzed the decay of the evanescent modes throughout an SC. The imaginary part of the wave number of the evanescent modes can be obtained experimentally with the measurements taken by 3DReAMS. These data represent the experimental confirmation of the analytical results obtained by the EPWE, as well as an experimental analysis of propagating and evanescent modes in an SC.

The paper is organized as follows. Sec. II summarizes the main ingredients of the PWE for 2D SCs with the explicit matrix formulation of the problem. In Sec. III we extend the PWE to the EPWE to solve the eigenvalue problem $k(\omega)$. We show the matrix formulation, as well as the EPWE, together with the supercell approximation for studying the complex band structures of 2D SC with point defects. In Sec. IV the complex band structures of an SC of polyvinyl chloride (PVC) cylinders embedded in air are obtained with EPWE for a 2D SC with, and without, point defects. Experimental results validating the predictions of the EPWE for the evanescent and propagating modes are shown in Sec. V. Finally, the work is summarized in Sec. VI.

\section{PLANE WAVE METHOD}

Propagation of sound is described by the equation

$$
\frac{1}{\rho c^{2}} \frac{\partial^{2} p}{\partial t^{2}}=\nabla\left(\frac{1}{\rho} \nabla p\right),
$$

where $c$ is the sound velocity, $\rho$ is the density of the medium, and $p$ is the pressure.

In this paper we consider a system composed of an array of straight, infinite cylinders made of an isotropic solid $A$, embedded in an acoustic isotropic background $B$. There is translational invariance in direction $z$ parallel to the cylinders' axis; and the system has a 2D periodicity in the transverse plane. By making use of this periodicity, we can expand the properties of the medium in the Fourier series,

$$
\sigma=\frac{1}{\rho(\vec{r})}=\sum_{\vec{G}} \sigma_{\vec{k}}(\vec{G}) e^{l \vec{G} \vec{r}}
$$

$$
\eta=\frac{1}{B(\vec{r})}=\sum_{\vec{G}} \eta_{\vec{k}}(\vec{G}) e^{l \vec{G} \vec{r}},
$$

where $\vec{G}$ is the 2D reciprocal-lattice vector and $B(\vec{r})$ $=\rho(\vec{r}) c(\vec{r})^{2}$ is the bulk modulus. For the pressure $p$ we use the Bloch theorem and harmonic temporal dependence,

$$
p(\vec{r}, t)=e^{l(\vec{k} \vec{r}-\omega t)} \sum_{\vec{G}} p_{k}(\vec{G}) e^{l \vec{G} \vec{r}} .
$$

It is simple to show that ${ }^{8}$

$$
\beta(\vec{G})=\left\{\begin{array}{lll}
\beta_{A} f+\beta_{B}(1-f) & \text { if } & \vec{G}=\overrightarrow{0} \\
\left(\beta_{A}-\beta_{B}\right) F(\vec{G}) & \text { if } & \vec{G} \neq \overrightarrow{0}
\end{array},\right.
$$

where $\beta=(\sigma, \eta)$ and $F(\vec{G})$ is the structure factor. For a circular cross section of radius $r$, the structure factor is

$$
F(\vec{G})=\frac{1}{A_{u c}} \int_{A_{c y l}} e^{-i \vec{G} \vec{r}} \overrightarrow{d r}=\frac{2 f}{G r} J_{1}(G),
$$

where $A_{u c}$ is the area of the unit cell, $A_{c y l}$ is the area of the cylinder, and $J_{1}$ is the Bessel function of the first kind of order 1.

Using Eqs. (1)-(4) we obtain ${ }^{8}$

$$
\begin{aligned}
& \sum_{\vec{G}^{\prime}}\left[(\vec{k}+\vec{G}) \sigma_{k}\left(\vec{G}-\vec{G}^{\prime}\right)\left(\vec{k}+\vec{G}^{\prime}\right)-\omega^{2} \eta\left(\vec{G}-\vec{G}^{\prime}\right)\right] p_{\vec{k}}\left(\vec{G}^{\prime}\right) \\
& \quad=0 .
\end{aligned}
$$

For $\vec{G}$ taking all the possible values, Eq. (7) constitutes a set of linear, homogeneous equations for the eigenvectors $p_{\vec{k}(\vec{G})}$, and eigenfrequencies $\omega(\vec{k})$. We obtain the band structures when $\vec{k}$ scans the area of the irreducible region of the first Brillouin zone.

Equation (7) can be expressed by the matrix formulation below

$$
\sum_{i=1}^{3} \Gamma_{i} \Sigma \Gamma_{i} P=\omega^{2} \Omega P,
$$

where $i=1,2$, and 3 . The matrices $\Gamma_{i}, \Sigma$, and $\Omega$ are defined as

$$
\left(\Gamma_{i}\right)_{m n}=\delta_{m n}\left(k_{i}+G_{i}^{m}\right) .
$$

The explicit matrix formulation is shown as follows:

$$
\begin{aligned}
\Gamma_{i} & =\left(\begin{array}{cccc}
k_{i}+G_{i} & 0 & \ldots & 0 \\
0 & k_{i}+G_{i} & \ldots & 0 \\
\vdots & \vdots & \ddots & \vdots \\
0 & \ldots & \ldots & k_{i}+G_{i}
\end{array}\right), \\
\Sigma & =\left(\begin{array}{ccc}
\sigma\left(\vec{G}_{1}-\vec{G}_{1}\right) & \ldots & \sigma\left(\vec{G}_{1}-\vec{G}_{N \times N}\right) \\
\vdots & \ddots & \vdots \\
\sigma\left(\vec{G}_{N \times N}-\vec{G}_{1}\right) & \ldots & \sigma\left(\vec{G}_{N \times N}-\vec{G}_{N \times N}\right)
\end{array}\right), \\
\Omega & =\left(\begin{array}{ccc}
\eta\left(\vec{G}_{1}-\vec{G}_{1}\right) & \ldots & \eta\left(\vec{G}_{1}-\vec{G}_{N \times N}\right) \\
\vdots & \ddots & \vdots \\
\eta\left(\vec{G}_{N \times N}-\vec{G}_{1}\right) & \ldots & \eta\left(\vec{G}_{N \times N}-\vec{G}_{N \times N}\right)
\end{array}\right),
\end{aligned}
$$




$$
P=\left(\begin{array}{c}
P\left(\vec{G}_{1}\right) \\
\vdots \\
P\left(\vec{G}_{N \times N}\right)
\end{array}\right),
$$

where $\vec{G}=\left(G_{x}, G_{y}, G_{x}\right)$. To solve (8) we must truncate the matrices. If we chose $m=n=(-M, \ldots, M)$, the size of the previous matrices is $N \times N=(2 M+1) \times(2 M+1) . N \times N$ is usually the number of plane waves used in the calculation.

By solving the system given in Eq. (8) for each Bloch vector in the irreducible area of the first Brillouin zone, we obtain $N \times N$ eigenvalues, $\omega^{2}$, which can be used to represent the band structures, $\omega(\vec{k})$.

\section{EXTENDED PLANE WAVE METHOD}

In the $\omega(\vec{k})$ formulation, the existence of BG is indicated by the absence of bands in determined ranges of frequencies. However, BG could be understood by means of the evanescent behavior of the internal modes. This interpretation was predicted by some authors ${ }^{23}$ when approximating the second band near the BG by expanding $\omega(\vec{k})$ to powers of $k$ around the edge $k=\pi / a$, being $a$ the lattice constant of the array. These authors claimed that as the BG is traversed, the exponential decay grows as the frequency nears the center of the BG. At a given frequency $\omega$ inside the BG, the evanescent wave is characterized by a complex value of its wave number $\vec{k}(\omega)$ and which the imaginary part characterizes as the exponential-like decay of the mode. In this section, we extend the previous PWE to the EPWE to obtain $\vec{k}(\omega)$ and with a possibly imaginary $k$.

From Eq. (8) we define the next vector,

$$
\Phi_{i}=\Sigma \Gamma_{i} P .
$$

With this definition we can reformulate the eigenvalue problem (8) as the equation system

$$
\begin{aligned}
& \Phi_{i}=\Sigma \Gamma_{i} P, \\
& \omega^{2} \Omega P=\sum_{i=1}^{3} \Gamma_{i} \Phi_{i} .
\end{aligned}
$$

To obtain an eigenvalue problem for $\vec{k}(\omega)$, we write $\vec{k}=k \vec{\alpha}$, where $\vec{\alpha}$ is a unit vector. Then Eq. (10) can be written as

$$
\Gamma_{i}=\Gamma_{i}^{0}+k \alpha_{i} I,
$$

where $I$ is the identity matrix, and

$$
\begin{aligned}
\Gamma_{i}^{0} & =\left(\begin{array}{cccc}
G_{i} & 0 & \ldots & 0 \\
0 & G_{i} & \ldots & 0 \\
\vdots & \vdots & \ddots & \vdots \\
0 & \ldots & \ldots & G_{i}
\end{array}\right), \\
\alpha_{i} & =\left(\begin{array}{cccc}
\alpha_{i} & 0 & \ldots & 0 \\
0 & \alpha_{i} & \ldots & 0 \\
\vdots & \vdots & \ddots & \vdots \\
0 & \ldots & \ldots & \alpha_{i}
\end{array}\right)
\end{aligned}
$$

Equation (8) can then be written as

$$
\begin{aligned}
& \left(\begin{array}{cc}
\omega^{2} \Omega-\sum_{i=1}^{3} \Gamma_{i}^{0} \Sigma \Gamma_{i}^{0} & 0 \\
-\sum_{i=1}^{3} \Sigma \Gamma_{i}^{0} & I
\end{array}\right)\left(\begin{array}{c}
P \\
\Phi^{\prime}
\end{array}\right)=k\left(\begin{array}{cc}
\sum_{i=1}^{3} \Gamma_{i}^{0} \Sigma \alpha_{i} & I \\
\sum_{i=1}^{3} \Sigma \alpha_{i} & 0
\end{array}\right) \\
& \times\left(\begin{array}{c}
P \\
\Phi^{\prime}
\end{array}\right),
\end{aligned}
$$

where $\Phi^{\prime}=\sum_{i=1}^{3} \alpha_{i} \Phi_{i}$.

Equation (19) represents a generalized eigenvalue problem with $2 N$ eigenvalues $k$ and possibly complex numbers for each frequency. Complex band structures have been calculated for the incidence direction characterized by vector $\vec{\alpha}$ by solving the previous eigenvalue equation for a discrete number of frequencies and then sorting by continuity of $k$. In contrast to the $\omega(\vec{k})$ method, the periodicity is not relevant in this formulation of the problem and $k(\omega)$ does not follow the first Brillouin zone.

Because of the periodicity of the system, Bloch waves can be expanded in a series of harmonics where each harmonic corresponds with a value of $k$, if $k$ is then a complex number, the evanescent behavior of a wave with a predetermined frequency would be multiexponential. ${ }^{15}$ The complex band structures show the values of all of the complex values of $k$ which contribute to the multiexponential decay of the mode in the BG. As we will see later, for the case of the SC analyzed in this paper, we can only approximate the evanescent behavior in the modes inside the BG by considering the first term of this harmonic expansion in terms of $k$.

\section{A. Supercell approximation}

One particularly interesting aspect of SCs is the possibility of creating point defects that confine acoustic waves in localized modes. ${ }^{17,22}$ Because of the locally breaking periodicity of the structure, defect modes can be created within the BG. These defect modes are strongly localized around the point defect: once the wave is inside the defect, it is trapped because the borders of the defect act as perfect mirrors for waves with frequencies in the BG. Localization depends on several parameters, such as the size of the point defect. However, in finite periodic structures the strength of sound localization also depends on the size of the structure around the defect because of the exponential decay of the outgoing wave. $^{14}$

To analyze the propagation of waves inside periodic structures with defects, authors have traditionally used PWE with supercell approximation. The supercell method requires the lowest possible interaction between defects. This results in a periodic arrangement of supercells that contain the point defect. With this method we can obtain the relation $\omega(\vec{k})$ for crystals with local defects and, for instance, the physics of wave guides ${ }^{13,18}$ or filters ${ }^{12}$ can be explained.

In this section, we apply the supercell approximation to the EPWE. This methodology enables us to obtain the relation $k(\omega)$ for defect modes. It will be interesting to discover how the imaginary part of the wave vector inside the BG changes with the creation of the defect. 
Consider an SC with primitive lattice vectors $\vec{a}_{i}(i$ $=1,2,3)$. The supercell is a cluster of $n_{1} \times n_{2} \times n_{3}$ scatterers periodically placed in space. The primitive lattice vectors in the supercell approximation are $\vec{a}_{i}^{\prime}=n_{i} \vec{a}_{i}$, and the complete set of lattices in the supercell approximation is $\left\{R^{\prime} \mid R^{\prime}\right.$ $\left.=l_{i} \vec{a}_{i}^{\prime}\right\}$, where $n_{i}$ and $l_{i}$ are integers. The primitive reciprocal vectors are then

$$
\vec{b}_{i}^{\prime}=2 \pi \frac{\varepsilon_{i j k} \vec{a}_{j}^{\prime} \times \vec{a}_{k}^{\prime}}{\vec{a}_{1}^{\prime} \cdot\left(\vec{a}_{2}^{\prime} \times \vec{a}_{3}^{\prime}\right)},
$$

where $\varepsilon_{i j k}$ is the completely antisymmetrical 3D Levi-Civita symbol. The complete set of reciprocal-lattice vectors in the supercell is $\left\{\vec{G} \mid \vec{G}_{i}=N_{i} \vec{b}_{i}^{\prime}\right\}$ where $N_{i}$ are integers.

Finally, the structural factor of the supercell in this approximation has to be computed while taking into account the size of the supercell. If we consider a 2D SC with cylindrical scatterers with a radius $r$ and an $n_{1} \times n_{2}$ sized supercell, the structure factor of the supercell is expressed by

$$
F(\vec{G})=\sum_{i=-\left(n_{1}-1\right) / 2}^{\left(n_{1}-1\right) / 2} \sum_{j=-\left(n_{2}-1\right) / 2}^{\left(n_{2}-1\right) / 2} e^{l\left(i a\left|\vec{G}_{1}\right|+j a\left|\vec{G}_{2}\right|\right) P(\vec{G})}
$$

where

$$
P(\vec{G})=\frac{2 f}{G r} J_{1}(G) .
$$

$f$ is the filling fraction of the supercell, $G=|\vec{G}|$, and $a$ is the lattice constant of the $2 \mathrm{D}$ periodic system.

By introducing the previous expressions in the matrices of the PWE [Eq. (8)] or in the case of the EPWE [Eq. (19)], we can then use the supercell approximation to calculate the band structure of a periodic structure with, and without, a point defect.

\section{NUMERICAL RESULTS}

We consider a 2D SC consisting of PVC cylinders of radius $r$ in an air background arranged in a square lattice with a lattice constant $a$. The material parameters employed in the calculations are $\rho_{\text {air }}=1.23 \mathrm{~kg} / \mathrm{m}^{3}, \quad \rho_{\mathrm{PVC}}$ $=1400 \mathrm{~kg} / \mathrm{m}^{3}, \quad c_{\text {air }}=340 \mathrm{~m} / \mathrm{s}$, and $c_{\mathrm{PVC}}=2380 \mathrm{~m} / \mathrm{s}$. We consider a filling fraction $f=\pi r^{2} / a^{2} \simeq 0.65$. We have used reduced magnitudes, ${ }^{8}$ so the reduced frequency is $\Omega$ $=w a /\left(2 \pi c_{\text {host }}\right)$ and the reduced wave vector is $K=k a /(2 \pi)$.

\section{A. Complete array}

In Fig. 1 we can observe the complex band structure obtained by EPWE for the SC described above. In the left panel we have represented the imaginary part of the wave vector in the $\Gamma X$ direction; in the right panel we have shown the complex band structures in the $\Gamma M$ direction; and the central panel shows the real part of the band structures. The imaginary part is not restricted in values of $k$; while the real part is restricted to the first Brillouin zone. The area in gray represents the full BG ranged between the frequencies $\Omega_{1}$ $=\omega_{1} a /\left(2 \pi c_{\text {host }}\right)=0.4057$ and $\Omega_{2}=\omega_{2} a /\left(2 \pi c_{\text {host }}\right)=0.7189$. Note that the real part of the complex band structures has exactly the same values as in the case of the PWE.

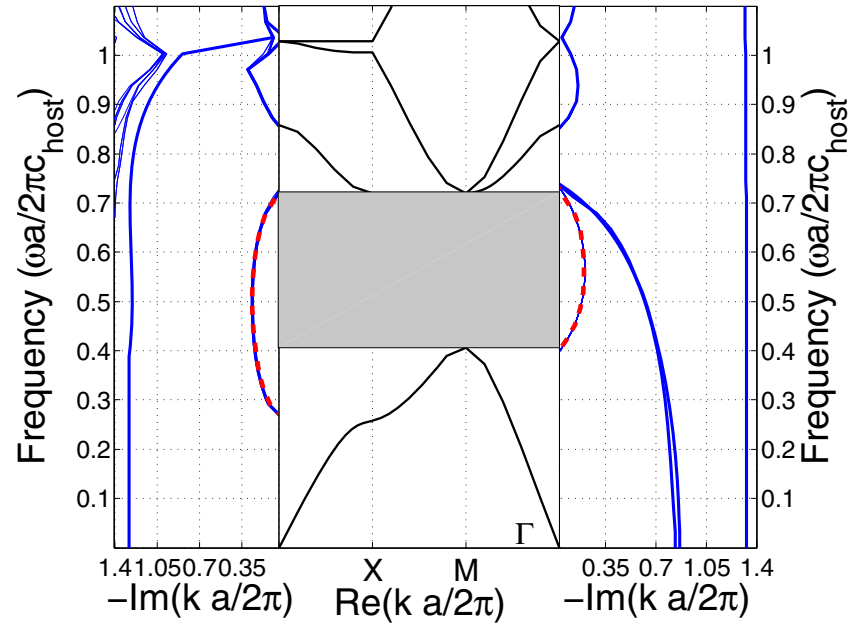

FIG. 1. (Color online) Band structure of an SC of PVC cylinders embedded in air with filling fraction $f \simeq 0.65$. The left panel represents the imaginary part of the wave vector for each $\Gamma X$ direction frequency. The central panel represents the real part of the wave vector, constrained in the first Brillouin zone, for each frequency. The right panel represents the imaginary part of the wave vector for each $\Gamma M$ direction frequency. The dashed line represents the imaginary part of the wave vector of the evanescent modes inside the BG. Reduced magnitudes have been used.

In Fig. 1 we can observe that modes inside the BG present purely imaginary wave vectors and these can be characterized as evanescent modes with an exponential-like decay. The elegant and intuitive explanation of the evanescent behavior of modes inside the $\mathrm{BG}$ given by Joannopoulus $^{23}$ is reproduced in Fig. 1 in $\Gamma X$; as well as in $\Gamma M$ directions (red dashed lines). The imaginary part of the wave number for frequencies inside the BG grows with values of frequency closer to the center of the BG; and disappears at the edges of the BG. In other words, the rate of decay is greater for frequencies closer to the center of the BG. We can also observe that the imaginary part of the wave vector connects propagating bands and so conserves the overall number of modes.

A recent paper has shown the multiexponential decay of evanescent modes in a photonic crystal. ${ }^{15}$ In Fig. 3, we can observe clearly that each frequency inside the BG is characterized by several values of $\operatorname{Im}(k)$, corresponding to the harmonics of the multiexponential decay of the evanescent modes. In the Sec. IV we will see that only the first value of the $\operatorname{Im}(k)$ contributes to the decay of the mode and, therefore, higher harmonics can be neglected and we can approximate in the same way as an exponential-like decay.

\section{B. Defect modes}

In this paper, point defects have been created by removing cylinders in an SC. We have used the EPWE method with supercell approximation to analyze the propagating and evanescent behavior of modes in an SC with point defects.

Figure 2 shows the complex band structures for the $\Gamma X$ direction and real band structures for an $\mathrm{SC}$ with a point defect. In our case, we use only one direction of incidence to analyze the complex band structure because the localized mode appears at the same frequency for all the incidence directions. The supercell used for the calculations is shown 


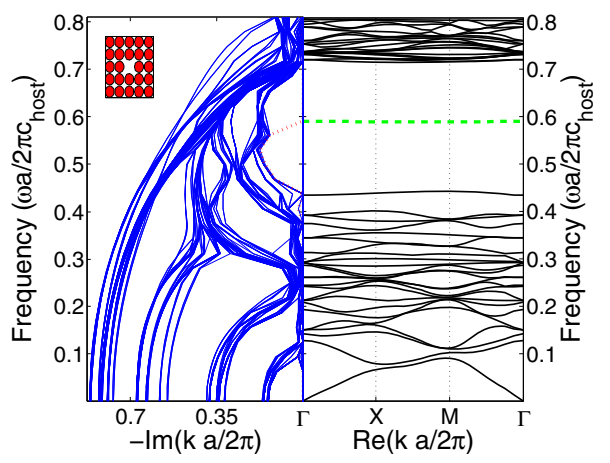

FIG. 2. (Color online) Band structure for an SC with an internal defect, calculated using the EPWE with supercell approximation. The left panel represents the imaginary part of the wave vector for each $\Gamma X$ direction frequency. The right panel represents the real part, constrained in the first Brillouin zone, of the wave vector for each frequency. The dashed line represents the frequency of the localized mode in the defect. The dotted line represents the imaginary part of the wave vector of the evanescent modes inside the BG. Reduced magnitudes have been used.

in the inset of Fig. 2. We can observe that the localized mode appears at $\Omega_{3}=\omega_{3} a /\left(2 \pi c_{\text {host }}\right)=0.59$ (green dashed line). For frequencies in the BG, the borders of the point defect act as perfect mirrors and produce the localized mode in this cavity. The complex value of the $k$ number for the modes inside the BG can be obtained by EPWE and becomes a purely real value for the localized mode (red dotted line and green dashed line). The value exactly coincides with the value obtained by PWE with supercell approximation.

\section{EXPERIMENTAL RESULTS}

We performed the experiments in an echo-free chamber sized $8 \times 6 \times 3 \mathrm{~m}^{3}$. To obtain the experimental dependence of the pressure all along the $\mathrm{SC}$, we measured the pressure field at several points between two rows of the SC. To achieve this we built a finite SC and placed the microphone inside the periodic structure in a space between two rows. The finite 2D SC used in this paper was made of PVC cylinders hung in a frame and measuring $5 a \times 5 a$. The radius of the cylinders was $r=10 \mathrm{~cm}$ and the lattice constant of the SC was $a=22 \mathrm{~cm}$. With these parameters, the finite $\mathrm{SC}$ has the same filling fraction $(f \simeq 0.65)$ as in Sec. IV and the dimensions are large enough for the microphone to be placed between the rows. The microphone used was a prepolarized free-field $1 / 2^{\prime \prime}$ Type $4189 \mathrm{~B} \& \mathrm{~K}$. The diameter of the microphone was $1.32 \mathrm{~cm}$, which is approximately $0.06 a$, and so a low level of influence over the pressure field measured is expected.

The 3DReAMS system is capable of sweeping the microphone through a 3D grid of measuring points located at any trajectory inside the echo-free chamber. The motion of the robot was controlled by an NI-PCI 7334 . We analyzed the absolute value of the sound pressure between two rows of the $\mathrm{SC}$ by moving the microphone in steps of $1 \mathrm{~cm}$.

In Sec. IV we analyzed the upper and lower frequencies of the BG for an SC of PVC cylinders with the filling fraction value as in our experimental setup. By considering the corresponding values of the parameters of our experimental $\mathrm{SC}$, we can obtain the frequency range of the BG. In our

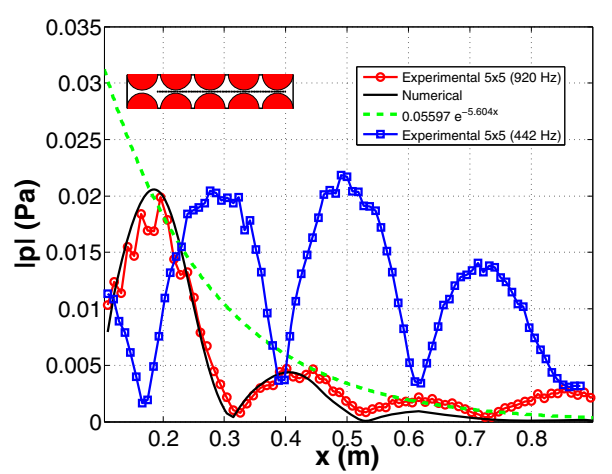

FIG. 3. (Color online) Absolute value of the pressure inside the SC in the positions between two rows. Squares represent these values for a frequency outside of the BG, $442 \mathrm{~Hz}$. Circles represent these values for a frequency inside the BG, $920 \mathrm{~Hz}$. Dashed line represents the fit of the exponential decay of the evanescent mode inside the BG. The continuous line represents the absolute values of the pressure obtained using finite element methods.

case, the BG appears between 627 and $1111 \mathrm{~Hz}$. To measure the propagation of sound inside the $\mathrm{SC}$, we analyzed two different frequencies, one inside the BG and the other in the first transmission band. The frequencies were $920 \mathrm{~Hz}$ and $442 \mathrm{~Hz}$, respectively.

In Fig. 3 we show the experimental measurements of the absolute value of the pressure inside SC for propagating and evanescent modes. These experimental results represent a novel measurement of the pressure field inside an SC. The inset of Fig. 3 shows the measured points in steps of $1 \mathrm{~cm}$ placed between two rows of cylinders inside the SC using the 3DReAMS system. Blue squares with a continuous blue polygonal line represent the absolute value of the pressure of a frequency outside of the BG, that is, $442 \mathrm{~Hz}$. This frequency represents a propagating mode inside the SC. Red circles with a polygonal red continuous line represent the absolute value of the pressure of a frequency inside the BG, that is, $920 \mathrm{~Hz}$. For the last case, we can observe the decay of the pressure inside the SC because of the evanescent behavior of the mode inside the BG.

In contrast to the propagating mode (blue squares with a blue polygonal continuous line), the evanescent mode (red squares with a red polygonal continuous line) is practically extinguished at the end of the crystal—and just a small value remaining for the emerging pressure. This characteristic of evanescent behavior in finite SCs has been measured recently by Wu et al. ${ }^{14}$ in an SC with a point defect.

The value of the imaginary part of the first harmonic of the wave vector for the $920 \mathrm{~Hz}$ frequency can be obtained from Fig. 1. Using the values of parameters of the $\mathrm{SC}$, we can observe a value $\operatorname{Im}(k)=-5.6 \mathrm{~m}^{-1}$. From experimental data (see Fig. 3), we can fit the decay of the evanescent mode. We have chosen the points with maximum values in order to fit an exponential decay $a e^{b x}$. The values of the fit are $a=0.05597 \pm 0.0103 \mathrm{~Pa}$ and $b=\operatorname{Im}(k)$ $=-5.60 \pm 1.45 \mathrm{~m}^{-1}$. Note that the experimental value is very close to the analytical value, i.e., the assumption that only the first harmonic is needed to represent the multiexponential decay of the evanescent mode is correct.

By solving the scattering problem inside the SC by means of the finite element method (FEM) we can analyze 
the evanescent behavior of the modes inside the BG of an SC. We have studied numerically the absolute value of the sound pressure between two rows of an SC. Continuity boundary conditions in the walls of the cylinders and the radiation condition at the borders of the numerical domain have been considered in the simulation. The black continuous line in Fig. 3 represents the absolute values of pressure obtained numerically inside the SC, considering an incidence of a plane wave with a frequency of $920 \mathrm{~Hz}$. The correspondence between the experimental data (red polygonal line with open red circles) and the numerical results is clear.

\section{CONCLUSIONS}

The propagation of waves inside periodic structures consists of propagating and evanescent modes. $\omega(\vec{k})$ methods can be used to analyze the propagating modes, while evanescent modes are represented by the absence of $k$ for some ranges of frequencies. In this paper, we extend the $\omega(\vec{k})$ to the $k(\omega)$ method for the case of 2D SCs. We present the formulation of the supercell approximation for the $k(\omega)$ method. For the EPWE we have predicted the evanescent nature of the modes inside the BG of an SC. In this paper we have reported measurements of the exponential-like decay of the acoustic field inside an SC. EPWE predicted a value for the imaginary part of the first harmonic of the wave number, $\operatorname{Im}(k)=-5.6 \mathrm{~m}^{-1}$; and by fitting an exponential decay, $a e^{b x}$, the experimental value we have obtained is $b=\operatorname{Im}(k)=$ $-5.60 \pm 1.45 \mathrm{~m}^{-1}$. Therefore, we can conclude that only the first harmonic contributes to the exponential-like decay of the evanescent mode. We have also shown that the imaginary part of the wave vector connects propagation bands and conserves the overall number of modes.

We have also applied the EPWE with supercell aproximation to SC with point defects. We have analyzed the case of one vacancy observing the localized mode inside the BG predicted by EPWE. The value of the $k$ number for this localized mode, that is purely imaginary in the case of complete SC, changes to purely real and it becomes in a passing mode as it was observed in the literature. The frequency of the localized mode exactly coincides with the value obtained by PWE.

Analytical, numerical, and experimental results reproduce with very good agreement the complex values of the wave vector inside the $\mathrm{BG}$, meaning that these methodolo- gies obtain good values for the exponential-like decay of the evanescent modes in an SC. This work shows the basis for the correct understanding of the design of narrow filters and wave guides based on PCs or SCs with point defects.

\section{ACKNOWLEDGMENTS}

The authors would like to thank Dr. E. A. Sánchez-Pérez for his comments and suggestions and thank Daniel Fenollosa and Talleres Ferriols for their help in building the mechanical part of 3DReAMS. This work was supported by MEC (Spanish government) and the European Regional Development Fund, under Grant Nos. MAT2009-09438 and MTM2009-14483-C02-02.

${ }^{1}$ R. Martínez-Sala, J. Sancho, J. V. Sánchez, V. Gómez, J. Llinares, and F. Meseguer, Nature 378, 241 (1995).

${ }^{2}$ M. S. Kushwaha, P. Halevi, L. Dobrzynski, and B. Djafari-Rouhani, Phys. Rev. Lett. 71, 2022 (1993)

${ }^{3}$ M. Sigalas and E. Economou, Solid State Commun. 86, 141 (1993).

${ }^{4}$ E. Yablonovitch, Phys. Rev. Lett. 58, 2059 (1987).

${ }^{5}$ S. John, Phys. Rev. Lett. 58, 2486 (1987).

${ }^{6}$ M. M. Sigalas, E. N. Economou, and M. Kafesaki, Phys. Rev. B 50, 3393 (1994).

${ }^{7}$ E. N. Economou and M. M. Sigalas, Phys. Rev. B 48, 13434 (1993).

${ }^{8}$ M. S. Kushwaha, P. Halevi, G. Martínez, L. Dobrzynski, and B. DjafariRouhani, Phys. Rev. B 49, 2313 (1994).

${ }^{9}$ H. Hernández-Cocoletzi, A. Krokhin, and P. Halevi, Phys. Rev. B 51, 17181 (1995).

${ }^{10}$ J. V. Sánchez-Pérez, D. Caballero, R. Mártinez-Sala, C. Rubio, J. SánchezDehesa, F. Meseguer, J. Llinares, and F. Gálvez, Phys. Rev. Lett. 80, 5325 (1998).

${ }^{11}$ J. V. Sánchez-Pérez, C. Rubio, R. Martínez-Sala, R. Sánchez-Grandia, and V. Gómez, Appl. Phys. Lett. 81, 5240 (2002).

${ }^{12}$ A. Khelif, A. Choujaa, B. Djafari-Rouhani, M. Wilm, S. Ballandras, and V. Laude, Phys. Rev. B 68, 214301 (2003).

${ }^{13}$ A. Khelif, M. Wilm, V. Laude, S. Ballandras, and B. Djafari-Rouhani, Phys. Rev. E 69, 067601 (2004).

${ }^{14}$ L. Wu, L. Chen, and C. Liu, Physica B 404, 1766 (2009).

${ }^{15}$ R. J. P. Engelen, D. Mori, T. Baba, and L. Kuipers, Phys. Rev. Lett. 102, 023902 (2009).

${ }^{16}$ F. Wu, Z. Hou, Z. Liu, and Y. Liu, Phys. Lett. A 292, 198 (2001).

${ }^{17}$ Y. Zhao and L. B. Yuan, J. Phys. D: Appl. Phys. 42, 015403 (2009).

${ }^{18}$ J. O. Vasseur, P. A. Deymier, B. Djafari-Rouhani, Y. Pennec, and A.-C. Hladky-Hennion, Phys. Rev. B 77, 085415 (2008).

${ }^{19}$ Y.-C. Hsue, A. J. Freeman, and B.-Y. Gu, Phys. Rev. B 72, 195118 (2005).

${ }^{20}$ V. Laude, Y. Achaoui, S. Benchabane, and A. Khelif, Phys. Rev. B 80, 092301 (2009).

${ }^{21}$ R. Sainidou and N. Stefanou, Phys. Rev. B 73, 184301 (2006).

${ }^{22}$ M. Sigalas, J. Appl. Phys. 84, 3026 (1998).

${ }^{23}$ J. D. Joannopoulus, S. G. Johnson, J. N. Winn, and R. D. Meade, Photonic Crystals: Molding the Flow of Light (Princeton University Press, Princeton, NJ, 2008). 